\title{
Accretion disks with a large scale magnetic field around black holes, and magnetic jet collimation
}

\section{G.S. Bisnovatyi-Kogan*}

Space Research Institute, Russian Academy of Sciences, Moscow, Russia

E-mail: gkogan@iki.rssi.ru

\section{R.V.E. Lovelace}

Cornell University, Ithaca, USA

E-mail: lovelacedastro.cornell.edu

\begin{abstract}
We discuss the problem of the formation of a large-scale magnetic field in the accretion disks around black holes, taking into account the nonuniform vertical structure of the disk. The high electrical conductivity of the outer layers of the disk prevents the outward diffusion of the magnetic field. This implies a stationary state with a strong magnetic field in the inner parts of the accretion disk close to the black hole, and zero radial velocity at the surface of the disk. Magnetic jet collimation is considered, when the jet radius is hold due to magneto-torsional oscillations. The range of parameters is found where jet radius is oscillating, regularly or chaotically, within restricted values.
\end{abstract}

25th Texas Symposium on Relativistic Astrophysics - TEXAS 2010

December 06-10, 2010

Heidelberg, Germany

${ }^{*}$ Speaker. 


\section{Introduction}

Quasars and AGN contain supermassive black holes, about 10 HMXR contain stellar mass black holes - microquasars. Jets are observed in objects with black holes: collimated ejection from accretion disks.

Early work on disk accretion to a black hole argued that a large-scale magnetic field of, for example, the interstellar medium would be dragged inward and greatly compressed by the accreting plasma [5, 6, 9]. Subsequently, analytic models of the field advection and diffusion in a turbulent disk suggested, that the large-scale field diffuses outward rapidly [12, 10], and prevents a significant amplification of the external poloidal field by electrical current in the accretion disk. This has led to the suggestion that special conditions (non-axisymmetry) are required for the field to be advected inward [14]. The question of the advection/diffusion of a large-scale magnetic field in a turbulent plasma accretion disk was reconsidered in [屯, taking into account its nonuniform vertical structure. The high electrical conductivity of the surface layers of the disk, where the turbulence is suppressed by the radiation flux and the relatively high magnetic field, prevents outward diffusion of the magnetic field. This leads to a strong magnetic field in the inner parts of accretion disks around black holes.

\section{The fully turbulent model}

There are two limiting accretion disk models which have analytic solutions for a large-scale magnetic field structure. The first was constructed in [5, 6] for a stationary non-rotating accretion disk. A stationary state in this disk (with a constant mass flux onto a black hole) is maintained by the balance between magnetic and gravitational forces, and thermal balance (local) is maintained by Ohmic heating and radiative conductivity for an optically thick conditions. The mass flux to the black hole in the accretion disk is determined by the finite conductivity of the disk matter and the diffusion of matter across the large-scale magnetic field as sketched in Fig.1. The value of the large-scale magnetic field in stationary conditions is determined by the accretion disk mass, which in turn is determined by the magnetic diffusivity of the matter. It is widely accepted that the laminar disk is unstable to different hydrodynamic, magnetohydrodynamic, and plasma instabilities which implies that the disk is turbulent. In X-ray binary systems the assumption about turbulent accretion disk is necessary for construction of a realistic models [13]. The turbulent accretion disks had been constructed also for non-rotating models with a large-scale magnetic field. A formula for turbulent magnetic diffusivity was derived in [6]. similar to the scaling of the shear $\alpha$-viscosity in turbulent accretion disk in binaries [13], where the viscous stress tensor component $t_{r \phi}=\alpha P$, with $\alpha \leq 1 \mathrm{a}$ dimensionless constant and $P$ the pressure in the disk midplane. disk. Using this representation, the expression for the turbulent electrical conductivity $\sigma_{t}$ is written as

$$
\sigma_{t}=\frac{c^{2}}{\tilde{\alpha} 4 \pi h \sqrt{P / \rho}} .
$$

Here, $\tilde{\alpha}=\alpha_{1} \alpha_{2}$. The characteristic turbulence scale is $\ell=\alpha_{1} h$, where $h$ is the half-thickness of the disk, the characteristic turbulent velocity is $v_{t}=\alpha_{2} \sqrt{P / \rho}$. The large-scale magnetic field threading a turbulent Keplerian disk arises from external electrical currents and currents in the 


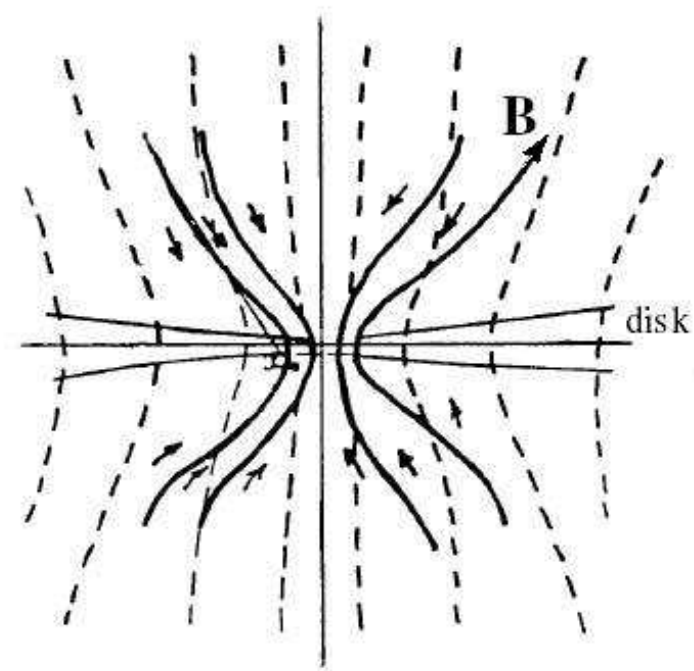

Figure 1: Sketch of the poloidal magnetic field threading an accretion disk. The field strength increases with decreasing radius owing to flux freezing in the accreting disk matter, from [6].

accretion disk. The field generated by the currents in the disk can be much larger than that due to the external currents. The magnetic field may become dynamically important, influencing the accretion disk structure and leading to powerful jet formation, if it is strongly amplified during the radial inflow of the disk matter. It is possible only when the radial accretion speed of matter in the disk is larger than the outward diffusion speed of the poloidal magnetic field due to the turbulent diffusivity $\eta_{t}=c^{2} /\left(4 \pi \sigma_{t}\right)$. Estimates in [12] have shown that for a turbulent conductivity (2.1), the outward diffusion speed is larger than the accretion speed. Thus it appears that there is no large-scale magnetic field amplification during Keplerian disk accretion. Numerical calculations in [12] are reproduced analytically for the standard accretion disk structure which can be written as (e.g.|(7)

$$
\dot{M}=4 \pi \rho v_{r} r h, h=\frac{v_{s}}{\Omega_{K}}, v_{s}=\sqrt{\frac{P}{\rho}}, 4 \pi r^{2} h \alpha P=\dot{M}\left(j-j_{i n}\right), \frac{3}{2} \frac{\Omega_{K}}{r}, \alpha \operatorname{Prh}=\frac{2 a T^{4} c}{3 \kappa \rho h} .
$$

Far from the inner disk boundary the specific angular momentum is $j \gg j_{i n}$. The characteristic time $t_{v i s c}$ of the matter advection due to the shear viscosity is $t_{v i s c}=\frac{r}{v_{r}}=\frac{j}{\alpha v_{s}^{2}}$. The time of the magnetic field diffusion is $t_{d i f f}=\frac{r^{2}}{\eta} \frac{h}{r} \frac{B_{z}}{B_{r}}, \eta=\frac{c^{2}}{4 \pi \sigma_{t}}=\tilde{\alpha} h v_{s}$. In the stationary state, the large-scale magnetic field in the accretion disk is determined by the equality $t_{v i s}=t_{\text {diff }}$, what determines the ratio

$$
\frac{B_{r}}{B_{z}}=\frac{\alpha}{\tilde{\alpha}} \frac{v_{s}}{v_{K}}=\frac{\alpha}{\tilde{\alpha}} \frac{h}{r} \ll 1
$$

Here, $v_{K}=r \Omega_{K}$ and $j=r v_{K}$ for a Keplerian disk. In a fully turbulent disk a matter is penetrating through magnetic field lines, almost without a field amplification. Note, that the field induced by the azimuthal disk currents has $B_{z d} \sim B_{r d}$ [1]. 

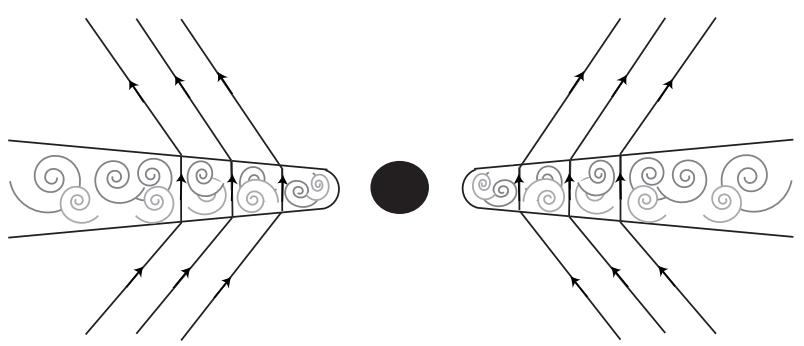

Figure 2: Sketch of the large-scale poloidal magnetic field threading a rotating turbulent accretion disk with a radiative outer boundary layer. The toroidal current flows mainly in the highly conductive radiative layers. The large-scale (average) field in the turbulent region is almost vertical.

\section{Turbulent disk with radiative outer zones}

Near the surface of the disk, in the region of low optical depth, the turbulent motion is suppressed by the radiative flux, similar to the suppression of the convection over the photospheres of stars with outer convective zones. The presence of the outer radiative layer does not affect the estimate of the characteristic time $t_{v i s c}$ of the matter advection in the accretion disk because it is determined by the main turbulent part of the disk. The time of the field diffusion, on the contrary, is significantly changed, because the electrical current is concentrated in the radiative highly conductive regions, which generate the main part of the magnetic field. The structure of the magnetic field with outer radiative layers is shown schematically in Fig.2. Inside the turbulent disk the electrical current is negligibly small so that the magnetic field there is almost fully vertical, with $B_{r} \ll B_{z}$, according to (2.3). In the outer radiative layer, the field diffusion is very small, so that matter advection is leading to strong magnetic field amplification. We suppose, that in the stationary state the magnetic forces could support the optically thin regions against gravity. When the magnetic force balances the gravitational force in the outer optically thin part of the disk of surface density $\Sigma_{p h}$ one finds the following relation takes place [6]

$$
\frac{G M \Sigma_{p h}}{r^{2}} \simeq \frac{B_{z} I_{\phi}}{2 c} \simeq \frac{B_{z}^{2}}{4 \pi}
$$

The surface density over the photosphere corresponds to a layer with effective optical depth close to $2 / 3$ (e.g. [1]). We estimate the lower limit of the magnetic field strength, taking $\kappa_{e s}$ (instead of the effective opacity $\left.\kappa_{e f f}=\sqrt{\kappa_{e s} \kappa_{a}}\right)$. Writing $\kappa_{e s} \Sigma_{p h}=2 / 3$, we obtain $\Sigma_{p h}=5 / 3\left(\mathrm{~g} / \mathrm{cm}^{2}\right)$ for the opacity of the Thomson scattering, $\kappa_{e s}=0.4 \mathrm{~cm}^{2} / \mathrm{g}$. The absorption opacity $\kappa_{a}$ is much less than $\kappa_{e s}$ in the inner regions of a luminous accretion disk. Thus using in equation (3.1), the above $\Sigma_{p h}$, we estimate the lower bound on the large-scale magnetic field of a Keplerian accretion disk as

$$
B_{z}=\sqrt{\frac{5 \pi}{3}} \frac{c^{2}}{\sqrt{G M_{\odot}}} \frac{1}{x \sqrt{m}} \simeq 10^{8} \mathrm{G} \frac{1}{x \sqrt{m}}, \quad x=\frac{r}{r_{g}}, \quad m=\frac{M}{M_{\odot}} .
$$

The maximum magnetic field is reached when the outward magnetic force balances the gravitational force on the disk of surface mass density $\Sigma_{p h}$. In equilibrium, $B_{z} \sim \sqrt{\Sigma_{p h}}$. We find that $B_{z}$ in a Keplerian accretion disk is about 20 times less than its maximum possible value, for $x=10, \alpha=0.1$, and $\dot{m}=10$. 


\section{Self-consistent numerical model}

Self-consistent models of the rotating accretion disks with a large-scale magnetic field requires solution the equations of magnetohydrodynamics. In presence of the radiative layer the strength of the magnetic field is large, and it may greatly exceed the strength of the seed field. The solution with a small field will not be stationary, and a transition to the strong field solution will take place. Therefore the strong field solution is the only stable stationary solution for a rotating accretion disk. The vertical structure of the disk with a large scale poloidal magnetic field was calculated in [11], taking into account the turbulent viscosity and diffusivity, and the fact that the turbulence vanishes at the surface of the disk. The full system of equations was reduced to one vertical equation for the non-dimensional radial velocity $u_{r}$, in the form

$$
\begin{gathered}
\alpha^{4} \beta^{2} \frac{\partial^{2}}{\partial \zeta^{2}}\left(g \frac{\partial}{\partial \zeta}\left(\tilde{\rho} g \frac{\partial}{\partial \zeta}\left(\frac{1}{\tilde{\rho}} \frac{\partial}{\partial \zeta}\left(\tilde{\rho} g \frac{\partial u_{r}}{\partial \zeta}\right)\right)\right)\right) \\
-\alpha^{2} \beta \mathscr{P} \frac{\partial^{2}}{\partial \zeta^{2}}\left(g \frac{\partial}{\partial \zeta}\left(\tilde{\rho} g \frac{\partial}{\partial \zeta}\left(\frac{u_{r}}{\tilde{\rho} g}\right)\right)\right)-\alpha^{2} \beta \mathscr{P} \frac{\partial^{2}}{\partial \zeta^{2}}\left(\frac{1}{\tilde{\rho}} \frac{\partial}{\partial \zeta}\left(\tilde{\rho} g \frac{\partial u_{r}}{\partial \zeta}\right)\right) \\
+\alpha^{2} \beta^{2} \frac{\partial^{2}}{\partial \zeta^{2}}\left(\tilde{\rho} g\left(u_{r}-g u_{0}\right)\right)+\mathscr{P}^{2} \frac{\partial^{2}}{\partial \zeta^{2}}\left(\frac{u_{r}}{\tilde{\rho} g}\right)+3 \beta \mathscr{P}^{2} \frac{u_{r}}{g}=0 .
\end{gathered}
$$

Here $\zeta \equiv z / h$ is a dimensionless height, $u_{r} \equiv-v_{r} /\left(\alpha c_{s 0}\right), u_{0}$ is a non-dimensional radial velocity in the non-magnetized disk [13]. Coefficients of the turbulent viscosity $v$, and magnetic diffusivity $\eta$ are connected by the magnetic Prandtl number $\mathscr{P} \sim 1, v=\mathscr{P} \eta=\alpha \frac{c_{s 0}^{2}}{\Omega_{K}} g(z)$, where $\alpha$ is a constant, determining the turbulent viscosity [13]; $\beta=c_{s 0}^{2} / v_{A 0}^{2}$, where $v_{A 0}=B_{0} /\left(4 \pi \rho_{0}\right)^{1 / 2}$ is the midplane Alfvén velocity, $\tilde{\rho}=\frac{\rho}{\rho_{0}}$. The function $g(z)$ accounts for the absence of turbulence in the surface layer of the disk [ $\llbracket$. In the body of the disk $g=1$, whereas at the surface of the disk, at say $z_{S}, g$ tends over a short distance to a very small value, effectively zero. The smooth function with a similar behavior is taken [11] in the form $g(\zeta)=\left(1-\frac{\zeta^{2}}{\zeta_{S}^{2}}\right)^{\delta}$, with $\delta \ll 1$. In the stationary state the boundary condition on the disk surface is $u_{r}=0$, and only one free parameter - magnetic Prandtl number $\mathscr{P}$ remains in the problem. In a stationary disk vertical magnetic field has a unique value. The example of the radial velocity distribution for $\mathscr{P}=1$ is shown in Fig.3.

\section{Jet collimation}

Magnetic collimation is connected with torsional oscillations of a cylinder with elongated magnetic field, see Fig.4. The stabilizing azimuthal magnetic field is created by torsional oscillations. Approximate simplified model is developed [2]. Ordinary differential equation is derived, and solved numerically, what gives a possibility to estimate quantitatively the range of parameters where jets may be stabilized by torsional oscillations.

In non-dimensional variables $\tau=\omega t, y=\frac{\tilde{R}}{R_{0}}, z=\frac{a \tilde{R}}{a_{0} R_{0}}, a_{0}=\frac{K}{\omega R_{0}^{2}}=\omega, R_{0}=\frac{\sqrt{K}}{\omega}$, differential equations have a form

$$
\frac{d y}{d \tau}=z, \frac{d z}{d \tau}=\frac{1}{y}\left(1-D \sin ^{2} \tau\right), y(0)=1, z=0 \text { at } \tau=0 .
$$




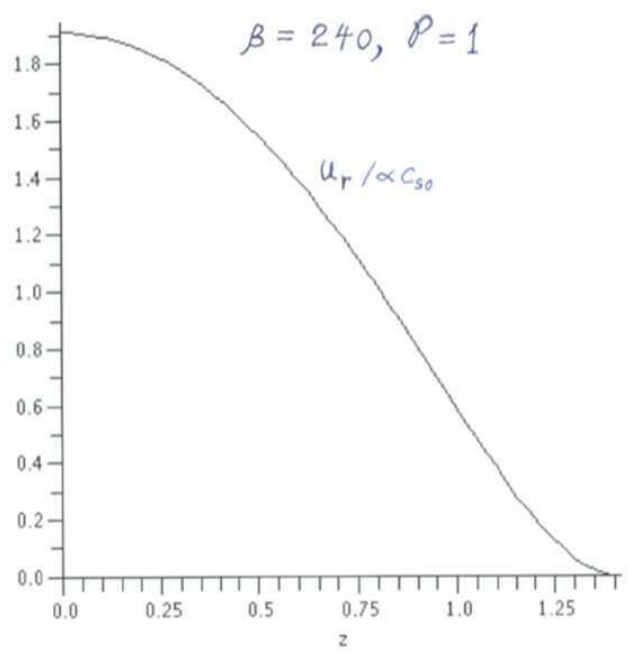

Figure 3: Distribution of the radial velocity over the thickness in the stationary accretion disk with a large scale poloidal magnetic field

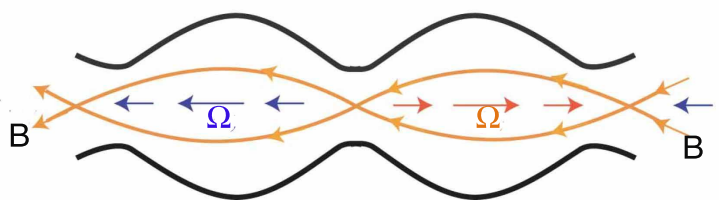

Figure 4: Jet confinement by magneto-torsional oscillations (qualitative picture)

The system (5.1) has two non-dimensional parameters: $D=\frac{1}{2 \pi K C_{m}}\left(\frac{C_{b} \Omega_{0}}{z_{0} \omega}\right)^{2}$, and $y(0)$, and the second one is taken equal to unity. Solution changes qualitatively with changing of the parameter $D$. At $D \leq 2$ there is no confinement, radius grows to infinity after several low-amplitude oscillations. At $D=2.1$ radius is not growing to infinity, but is oscillating around some average value, forming complicated curves (Fig. 5).At $D \geq 2.28$ the radius goes to zero. On the edge of the cylinder the rotational velocity cannot exceed the light velocity. The analysis have shown [2] that for the sound velocity not exceeding $c / 2$, the jet should contain baryons, density $\rho_{0}$ exceeding about $30 \%$ of the total density of the jet. Development of chaos in this system, by construction of Poincare sections was investigated in [8].

\section{Conclusions}

1. Disk field is amplified during disk accretion due to high conductivity in outer radiative layers. Stationary solution corresponds to $\beta=240$ for $\operatorname{Pr}=1$.

2. Jets from accretion disk are magnetically collimated by large scale poloidal magnetic field by torsion oscillations, which may be regular or chaotic. 

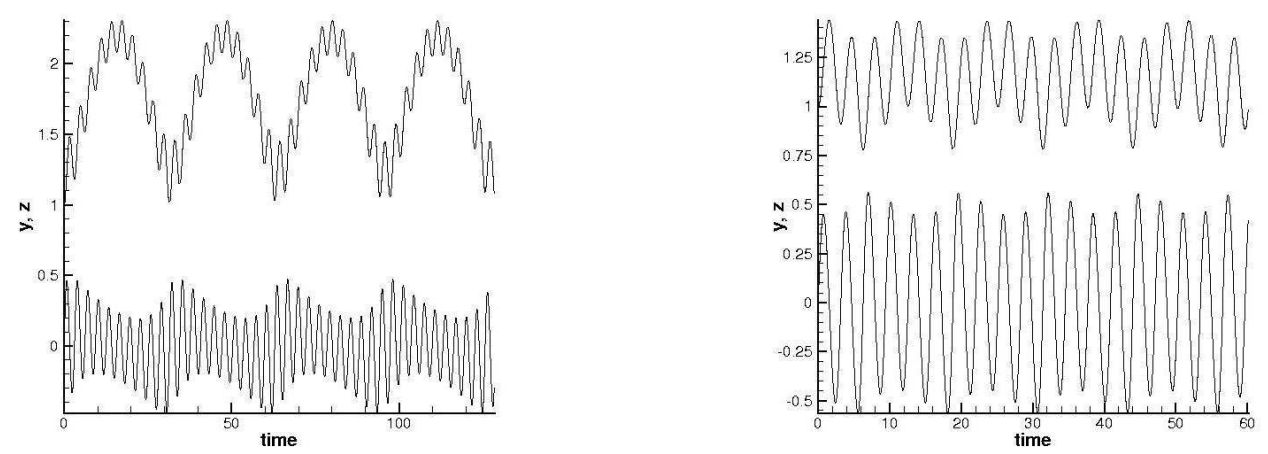

Figure 5: Time dependence of non-dimensional radius $y$ (upper curve), and non-dimensional velocity $z$ (lower curve), for $D=2.11$ (left); for $D=2.25$ (right), from [2].

\section{References}

[1] G.S. Bisnovatyi-Kogan, Stellar physics. Vol.1,2 (2001) Berlin: Springer

[2] G.S. Bisnovatyi-Kogan, Dynamic confinement of jets by magnetotorsional oscillations, MNRAS 376 (2007) 457

[3] G.S. Bisnovatyi-Kogan, \& S.I. Blinnikov, The Equilibrium, Stability and Evolution of a Rotating Magnetized Gaseous Disk, Ap\&SS 19 (1972) 93

[4] G.S. Bisnovatyi-Kogan, \& R.V.E. Lovelace, Large-Scale B-Field in Stationary Accretion Disks, ApJL 667 (2007) L167

[5] G.S. Bisnovatyi-Kogan, \& A.A. Ruzmaikin, The Accretion of Matter by a Collapsing Star in the Presence of a Magnetic Field, Ap\&SS 28 (1974) 45

[6] G.S. Bisnovatyi-Kogan, \& A.A. Ruzmaikin, The accretion of matter by a collapsing star in the presence of a magnetic field. II - Selfconsistent stationary picture, Ap\&SS 42 (1976) 401

[7] G.S. Bisnovatyi-Kogan, \& R.V.E. Lovelace, Advective accretion disks and related problems including magnetic fields, New Astron. Rev. 45 (2001) 663

[8] G.S. Bisnovatyi-Kogan, A.I. Neishtadt, Z.F. Seidov, O.Yu. Tsupko, \& Yu.M. Krivosheev, Dynamical chaos in the problem of jet collimation, MNRAS (submitted) (2011)

[9] R.V.E. Lovelace, Dynamo model of double radio sources, Nature 262 (1976) 649

[10] R.V.E. Lovelace, M.M. Romanova, \& W.I. Newman, Implosive accretion and outbursts of active galactic nuclei, ApJ 437 (1994) 136

[11] R.V.E. Lovelace, D.M. Rothstein, G.S. Bisnovatyi-Kogan, Advection/Diffusion of Large-Scale B Field in Accretion Disks, ApJ 701 (2009) 885

[12] S.H. Lubow, J.C.B. Papaloizou, \& J.E. Pringle, Magnetic field dragging in accretion discs, MNRAS 267 (1994) 235

[13] N.I. Shakura, \& R.A. Sunyaev, Black holes in binary systems. Observational appearance., AAp 24 (1973) 337

[14] H.C. Spruit,\& D.A. Uzdensky, Magnetic Flux Captured by an Accretion Disk, ApJ 629 (2005) 960 